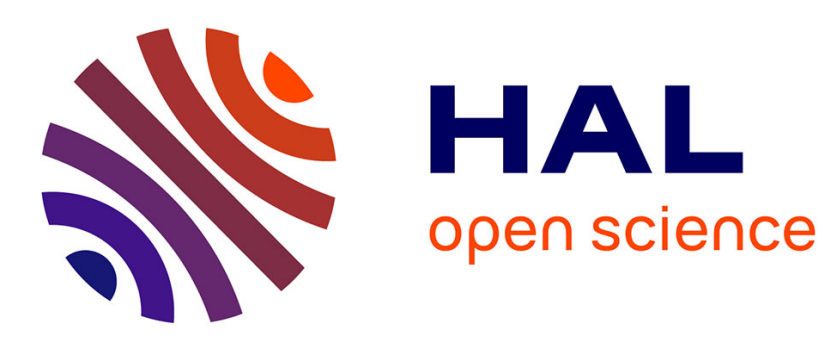

\title{
Swinging of Red Blood Cells under Shear Flow
}

Manouk Abkarian, Magalie Faivre, Annie Viallat

\section{To cite this version:}

Manouk Abkarian, Magalie Faivre, Annie Viallat. Swinging of Red Blood Cells under Shear Flow. Physical Review Letters, 2007, 98 (18), 10.1103/physrevlett.98.188302 . hal-01870703

\section{HAL Id: hal-01870703 https://hal.science/hal-01870703}

Submitted on 9 Sep 2018

HAL is a multi-disciplinary open access archive for the deposit and dissemination of scientific research documents, whether they are published or not. The documents may come from teaching and research institutions in France or abroad, or from public or private research centers.
L'archive ouverte pluridisciplinaire HAL, est destinée au dépôt et à la diffusion de documents scientifiques de niveau recherche, publiés ou non, émanant des établissements d'enseignement et de recherche français ou étrangers, des laboratoires publics ou privés. 


\title{
Swinging of Red Blood Cells under Shear Flow
}

\author{
Manouk Abkarian, ${ }^{1,3, *}$ Magalie Faivre, ${ }^{2,3}$ and Annie Viallat ${ }^{2,3, \dagger}$ \\ ${ }^{1}$ Laboratoire des Colloides, Verres et Nanomatériaux, UMR 5587, CNRS/UM2, CC26, 34095 Montpellier Cedex 5, France \\ ${ }^{2}$ Laboratoire Adhésion et inflammation, Inserm U600/CNRS UMR 6212 Université de la Méditerranée, Case 937, 163 Avenue de \\ Luminy, 13288 Marseille Cedex 9, France \\ ${ }^{3}$ Laboratoire de Spectrométrie Physique, UMR 5588 CNRS/UJF, BP 87, 38402 Saint Martin d'Hères, France
}

(Received 27 June 2006; published 30 April 2007)

\begin{abstract}
We reveal that under moderate shear stress $(\eta \dot{\gamma} \approx 0.1 \mathrm{~Pa}$ ) red blood cells present an oscillation of their inclination (swinging) superimposed to the long-observed steady tank treading (TT) motion. A model based on a fluid ellipsoid surrounded by a viscoelastic membrane initially unstrained (shape memory) predicts all observed features of the motion: an increase of both swinging amplitude and period $(1 / 2$ the TT period) upon decreasing $\eta \dot{\gamma}$, a $\eta \dot{\gamma}$-triggered transition toward a narrow $\eta \dot{\gamma}$ range intermittent regime of successive swinging and tumbling, and a pure tumbling at low $\eta \dot{\gamma}$ values.
\end{abstract}

DOI: 10.1103/PhysRevLett.98.188302

PACS numbers: 83.80.Lz, 83.50.- -v, 87.17.Jj

A human red blood cell (RBC) is a biconcave diskshaped membrane encapsulating a Newtonian solution of hemoglobin. The membrane is composed by a fluid incompressible lipid bilayer underlined by a thin elastic cytoskeleton [1]. This complex structure determines the RBC behavior in shear flow, which greatly influences flow and mass transport in the microcirculation in both health and disease [2]. However, this behavior is not well understood yet and important questions remain open. First, the state of deformation of the elastic membrane at rest is still debated. Does RBC present shape memory (membrane elements of the rim and the dimples nonmechanically equivalent) as recently suggested [3]? Second, flowing RBCs were observed [4-7] only when the cells were suspended in plasma and present an unsteady tumbling (T) solidlike motion [4] or when they are subjected to a high shear stress and exhibit a droplike tank treading (TT) motion characterized by a steady orientation and membrane rotation about the internal fluid. The RBC regime of motion at smaller shearstress and close to the T-TT transition has not been studied, although it is of crucial importance. Indeed, the simplest models, which treat RBCs like fluid ellipsoids [7-9] retrieve T and TT motions but do not capture the shear-rate dependency of the transition, thus raising the question of the role of the elasticity of the membrane on the cell behavior.
Here, by using a recent method of cell imaging parallel to the shear plane [10], we explore the RBC movement close to the T-TT transition. In the TT regime, we reveal that RBCs present an oscillation of their inclination with a period equal to half the TT period that we name swinging (S). We characterize the shear-stress dependence of this oscillation down to the T-TT transition. We show that the transition to pure $\mathrm{T}$ is preceded by a narrow critical shearstress regime where the RBC exhibits an intermittent $\mathrm{S}$-T behavior. Finally, we propose a model, assuming an elastic nonspherical RBC membrane, which captures the main features of the observed behavior.

Materials and methods. - Direct measurements of the orientation of the cells with respect to the flow direction (angle $\theta$ ) and cell shape (lengths of the long and small axis of the cell cross section, $a_{1}$ and $a_{2}$, respectively) are provided from side-view microscopic imaging [10] [Fig. 1(a)]. We varied the wall shear rate $\dot{\gamma}$ (in the range $0-5 \mathrm{~s}^{-1}$ ) and the outer viscosity $\eta_{0}$ by suspending RBCs in various solutions of dextran (concentration $6 \%, 7.5 \%$, or $9 \% \mathrm{w} / \mathrm{w}$ and viscosity 22,31 , and $47 \mathrm{mPa} \cdot \mathrm{s}$, respectively). Correspondingly, the wall shear stress, $\eta_{0} \dot{\gamma}$, varies in a range from 0 to $0.25 \mathrm{~Pa}$. We observed (i) the motion of flowing RBCs at a fixed $\eta_{0} \dot{\gamma}$, (ii) the motion of individual cells at various $\eta_{0} \dot{\gamma}$ (for $8 \mathrm{RBCs}$ ), and (iii) the T-TT transition of 35 cells by increasing and/or decreasing $\eta_{0} \dot{\gamma}$.

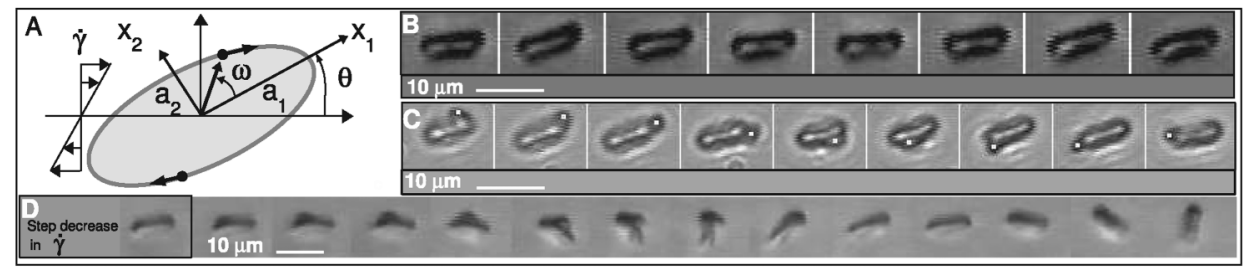

FIG. 1. Units $\left[\eta_{i}\right]=\left[\eta_{0}\right]=\left[\eta_{m}\right]=\mathrm{mPa} \cdot \mathrm{s},[\mu]=\mathrm{Pa}$ and $[\dot{\gamma}]=\mathrm{s}^{-1}$. (a) Schematic drawing of a TT ellipsoid in a shear flow. (b) RBC swinging $\left(\dot{\gamma}=1.33, \eta_{0}=47\right)$. Time sequence of $2 \mathrm{~s}$. (c) Rotation of a bead (diameter $\left.1 \mu \mathrm{m}\right)$ stuck on the membrane of a RBC with $\left(\dot{\gamma}=6, \eta_{0}=47\right)$. Time sequence of $1 \mathrm{~s}$. (d) The transition from $\mathrm{S}$ to $\mathrm{T}$ induced by decreasing $\dot{\gamma}$ is associated with a transient localized deformation ( $\left.\eta_{0}=47, \dot{\gamma}=2.66\right)$. Time sequence of $1 \mathrm{~s}$. 

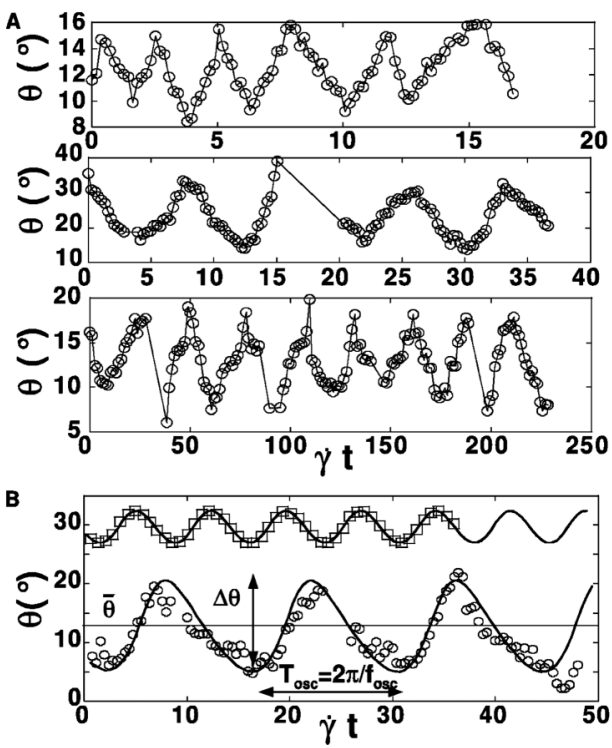

FIG. 2. Same units as in Fig. 1. (a) Orientation versus the normalized time $\dot{\gamma} t$ for various cells from top to bottom: $(\dot{\gamma}=$ $\left.1.8, \quad \eta_{0}=22\right), \quad\left(\dot{\gamma}=2.6, \quad \eta_{0}=31\right), \quad\left(\dot{\gamma}=6.6, \quad \eta_{0}=47\right)$. (b) Orientation versus $\dot{\gamma} t$ for $(\bigcirc)$ a RBC with $\left(\dot{\gamma}=0.8, \eta_{0}=\right.$ 47), solid line from Eq. (2) with $\left(\eta_{m}=1120, \mu_{m}=0.42\right)$; ( $\square$ ) a polymeric capsule from [18] with $\left(\dot{\gamma}=18, \eta_{0}=964\right)$. Solid line from Eqs. (2) with the surface moduli $\left(\eta_{m} e=0.085 \mathrm{mPa} \cdot\right.$ $\left.\mathrm{s} / \mathrm{m}, \mu_{m} e=0.675 \mathrm{mPa} \cdot \mathrm{m}\right)$, and $\left(a_{1}=278.8 \mu \mathrm{m}, a_{2}=a_{3}=\right.$ $170.8 \mu \mathrm{m})$ obtained from the size at rest $R_{0}=224.8 \mu \mathrm{m}$ of the capsule and its mean deformation during flow: $D=$ $\left(a_{1}-a_{2}\right) /\left(a_{1}+a_{2}\right) \approx 0.12$ at $\dot{\gamma}=18$.

Observations. - A typical behavior is illustrated in Fig. 1. For high $\eta_{0} \dot{\gamma}$ values, RBCs present a quasisteady TT motion as previously reported [5-7]. When $\eta_{0} \dot{\gamma}$ is decreased, the RBCs inclination oscillates about a mean angle (Fig. 1 and [11]), down to a critical $\eta_{0} \dot{\gamma}_{c}^{-}$, for which RBCs tumble at least once [Fig. 1(d)]. Swinging is characterized by a quasi-nondeformed cell shape (maximum variation of $a_{1} \leq 5 \%$ ) and a mean value of the cell inclination ranging from $6^{\circ}$ to $25^{\circ}$ [Figs. 1(b) and 2]. Moreover, as revealed from observing small carboxylated beads stuck to the membrane [Fig. 1(c)], S occurs while the membrane tank treads and the oscillation period is equal to half the TT period. Observations of single RBCs at various $\dot{\gamma}$ show that both the magnitude $\Delta \theta=\theta_{\max }-\theta_{\min }$ and the period $T_{\text {osc }}$ of oscillation increase significantly upon decreasing $\dot{\gamma}$ as illustrated in Figs. 3(a) and 3(b). The transition of movement from pure T to pure $\mathrm{S}$ (respectively, pure $\mathrm{S}$ to pure $\mathrm{T}$ ) is induced by tuning up (respectively down) $\dot{\gamma}$. Its more striking feature is the existence of a regime of intermediate motion where the cells alternatively present tumbles of $180^{\circ}$ separated by several S oscillations [Fig. 4(a)]. Given the experimental constraints, it is not easy to follow the cells long enough to observe a large series of tumbles and swings to determine the $\dot{\gamma}$ domain of intermittency. We choose to observe the shear rate corresponding to a change in movement from $\mathrm{S}$ to $\mathrm{T}\left(\dot{\gamma}_{c}^{<}\right)$with decreasing $\dot{\gamma}$, and from T to $\mathrm{S}\left(\dot{\gamma}_{c}^{>}\right)$with increasing $\dot{\gamma}$ over a time scale of $\sim 20 \mathrm{~s}$.

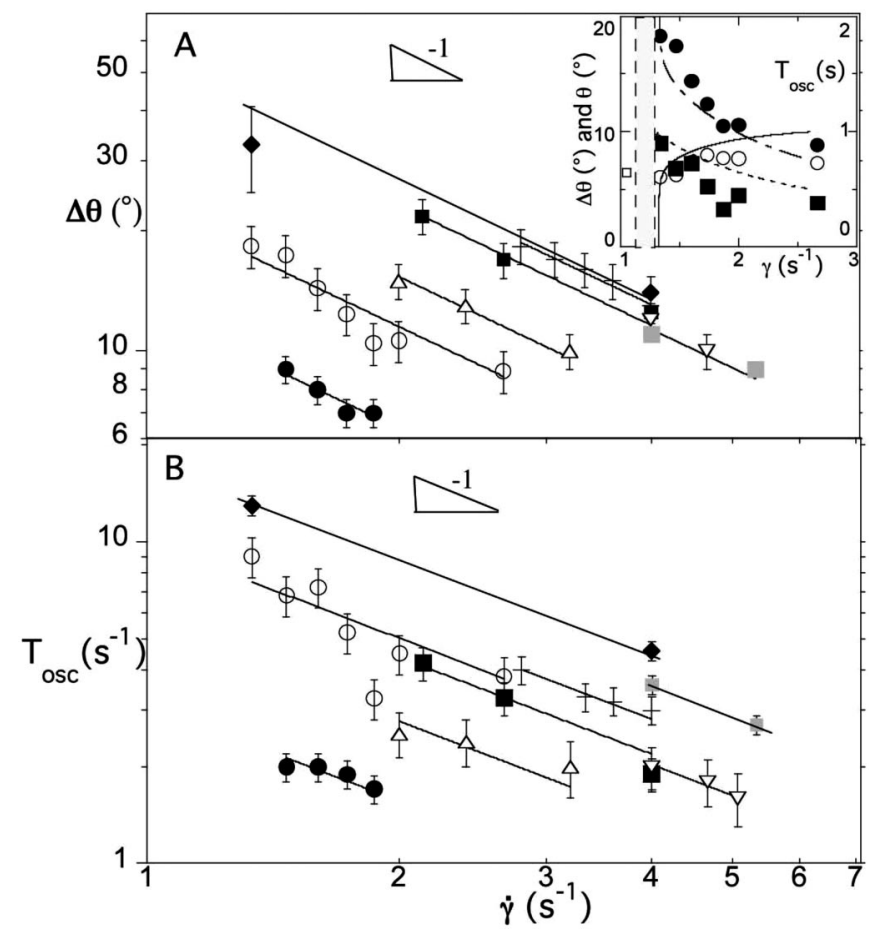

FIG. 3. Same units as in Fig. 1. Experimental $\dot{\gamma}$ variations of (a) $\Delta \theta$ and (b) $T_{\text {osc }}$ on single RBCs: $\eta_{0}=22:(\bigcirc),(\bigcirc)$ : cell (a); $\eta_{0}=31:(\square),(\square) ; \eta_{0}=47:(\diamond),(\triangle),(+),(\nabla)$. (a) inset for cell (a): (@) $\Delta \theta,(\square) T_{\text {osc }},(\square)$ one tumbling period value, and $(\bigcirc) \bar{\theta}$ versus $\dot{\gamma}$. Corresponding curves of the model with $\mu_{m}=0.38$ and $\eta_{m}=700$ : (dash-dotted line) $\Delta \theta$, (dashed line) $T_{\mathrm{osc}}$, and (solid line) $\bar{\theta}$.

The difference $\dot{\gamma}_{c}^{>}-\dot{\gamma}_{c}^{<}$, that we call hysteresis, is considered to give an order of magnitude of the intermittency $\dot{\gamma}$ domain: for instance for two different RBCs at $\eta_{0}=$ $31 \mathrm{mPa} \cdot \mathrm{s}, \dot{\gamma}_{c}^{<}=0.47 \mathrm{~s}^{-1}$ while $\dot{\gamma}_{c}^{>}=1 \mathrm{~s}^{-1}$ and $\dot{\gamma}_{c}^{<}=$ $0.8 \mathrm{~s}^{-1}$ while $\dot{\gamma}_{c}^{>}=1.73 \mathrm{~s}^{-1}$, respectively. Distribution functions of $\eta_{0} \dot{\gamma}_{c}^{>}$and $\eta_{0} \dot{\gamma}_{c}^{<}$obtained on 35 RBCs are shown in Fig. 4(b). The hysteresis, estimated from the distance between the two maxima of the two distributions, is of the order of $10^{-3} \mathrm{~Pa}$.

Swinging and previous studies on RBCs. - Previous studies, performed at higher $\eta_{0} \dot{\gamma}$ did not detect cell oscil-
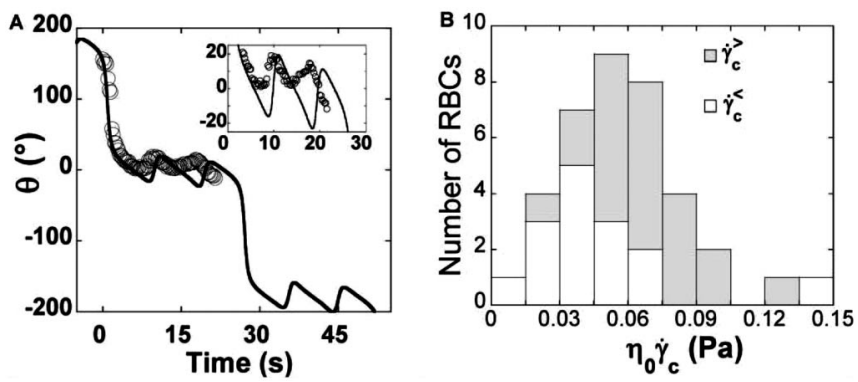

FIG. 4. Same units as in Fig. 1. (a) (O) Successive $\mathrm{S}$ and $\mathrm{T}$ at $\left(\eta_{0}=22, \quad \dot{\gamma}=1.526\right)$; (solid line) numerical calculus with $\left(\eta_{0}=22, \dot{\gamma}=1.526, \mu_{m}=0.454, \eta_{m}=700\right)$. (b) Distribution function of critical shear stresses of transition for increasing $\left(\dot{\gamma}^{>}\right)$and decreasing $\left(\dot{\gamma}^{<}\right)$shear rates. 
lation, likely because cells were observed perpendicularly to the shear plane. Nevertheless, we report in [11] the $\dot{\gamma}$ variations of the oscillation frequency $f_{\text {osc }}$, obtained in this study together with the TT frequencies $\dot{\omega}$ reported in the literature for the higher $\eta_{0} \dot{\gamma}[6,7]$. The two sets of points are almost overlapping only if $f_{\text {osc }}$ is divided by 2 . This illustrates again the direct link between membrane rotation and oscillation.

Swinging and previous studies on elastic shells. - The full RBC behavior we described here has never been observed on purely viscous objects such as lipid vesicles $[10,12]$ nor predicted $[8,13-15]$. Oscillations have, however, been detected on systems that differ from RBCs by their small nonsphericity at rest and the high extensibility of their surface, namely, elastic protein-coated drops [16] and polymer capsules $[17,18]$. In the latter case, the factor of 2 between TT and oscillation periods and the $\Delta \theta$ decrease with increasing $\dot{\gamma}$ were observed but no transition to tumbling was found. Moreover, no explanation of the oscillation was proposed, neither by these authors nor by Ramanujan and Pozrikidis [19], who retrieved a shell oscillation (limited to one period) from a full numerical simulation performed on a TT elastic biconcave shell.

Model. - We propose here a simple analytical approach based on the classical framework of Keller and Skalak (KS) [7-9], which treat the RBC as a fluid ellipsoidal membrane enclosing a viscous liquid. The $\mathrm{KS}$ model qualitatively retrieves TT and T, but predict neither the shear-rate dependency of the transition, nor the $\mathrm{S}$ behavior. Notably, they do not account for the elastic energy storage, which may be induced by local deformations of the cytoskeleton during TT. Indeed, if one assumes RBC shape memory, the local elements of the composite membrane (cytoskeleton and lipid bilayer), including the elements which form the rim and the dimples, are not equivalent and are not strained in the biconcave resting shape. During TT, the elements which form the rim at rest rotate about the stationary cell shape to reach the dimples after rotation and reciprocally. They are then locally strained and store elastic energy. After a $\pi$ rotation, the elements retrieve their initial shape and are no more strained. It is clear that this periodic storage of energy is significant only when the unstrained $\mathrm{RBC}$ shape is nonspherical, otherwise the membrane elements tank tread without modifying the global state of stress of the cell, preserving the steady nature of the TT motion. In order to derive tractable equations of motion, we consider an oblate ellipsoid filled with a viscous liquid and delimited by a viscoelastic 3D thin membrane, which includes the lipid bilayer and the underlying cytoskeleton [20]. The membrane elements are prescribed to rotate along elliptical trajectories parallel to the shear plane, with a linear velocity field given by $v_{1}=-\dot{\omega}\left(a_{1} / a_{2}\right) x_{2}$, $v_{2}=\dot{\omega}\left(a_{2} / a_{1}\right) x_{1}, v_{3}=0$, where $\omega$ and $\dot{\omega}$ are the phase angle of a membrane element and its instantaneous frequency of tank treading, respectively. The KS equation for $\mathrm{RBC}$ motion is obtained by stating that at equilibrium the total moment exerted by the external fluid on the cell vanishes [first equation in Eqs. (2)]. In addition, the movement satisfies the conservation of energy; i.e., the rate of dissipation of energy in the cell must equal the rate at which work is done by the external fluid on the cell. KS calculated both rates assuming viscous energy dissipation in the cell. We add to this latter contribution the elastic power stored in the periodic elastic strain of the cytoskeleton [22]: $P_{\mathrm{el}}=\int_{\Omega} \operatorname{Tr}(\sigma: \mathbf{D}) d \Omega$, where $\Omega$ is the membrane volume, $\mathbf{D}$ the Eulerian strain rate tensor derived from the KS velocity field, and $\sigma$ the shear-stress tensor in the membrane; $\sigma$ is computed from the local deformation of the membrane due to TT, assuming a simple KelvinVoigt viscoelastic material: $\sigma=2 \eta_{m} \mathbf{D}+2 \mu_{m} \mathbf{E}$, where $\mathbf{E}$ is the Euler-Almansi strain tensor obtained from the KS velocity field. After some algebra, $P_{\text {el }}$ writes as

$$
P_{\mathrm{el}}=\frac{1}{2} \dot{\omega}\left(\frac{a_{2}}{a_{1}}-\frac{a_{1}}{a_{2}}\right)^{2}\left[2 \eta_{m} \dot{\omega}+\mu_{m} \sin (2 \omega)\right] \Omega,
$$

where $\eta_{m}$ and $\mu_{m}$ are the membrane viscosity and the shear modulus, respectively. Conservation of energy provides a constraint on the allowable RBC motion and yields a second differential equation (for more details, see [11]). The two coupled equations are

$$
\begin{aligned}
\frac{\dot{\theta}}{\dot{\gamma}}= & -\frac{1}{2}-\frac{2 a_{1} a_{2}}{a_{1}^{2}+a_{2}^{2}} \frac{\dot{\omega}}{\dot{\gamma}}+\frac{1}{2} \frac{a_{1}^{2}-a_{2}^{2}}{a_{1}^{2}+a_{2}^{2}} \cos (2 \theta), \\
\frac{\dot{\omega}}{\dot{\gamma}}= & \frac{-f_{3}}{f_{2}-\frac{\eta_{i}}{\eta_{0}}\left(1+\frac{\eta_{m}}{\eta_{i}} \frac{\Omega}{V}\right) f_{1}} \\
& \times\left[\cos (2 \theta)-\frac{f_{1}}{2 f_{3}} \frac{\mu_{m}}{\eta_{0} \dot{\gamma}} \frac{\Omega}{V} \sin (2 \omega)\right],
\end{aligned}
$$

where $\dot{\theta}$ is the time derivative of the cell inclination, $f_{1}, f_{2}$, and $f_{3}$ are geometrical constants, and $V$ is the RBC volume (same definition as in [8]). The limiting case $\mu_{m}=\eta_{m}=$ 0 corresponds to KS. In order to test whether this simplified model retrieves the observed RBC behavior, we numerically solved the equations using the following set of parameters: $a_{1}=a_{3}=4 \mu \mathrm{m}, a_{2}=1.5 \mu \mathrm{m}, \Omega=\Sigma e$ [23], where $\Sigma$ is the oblate ellipsoid area and $e=50 \mathrm{~nm}$ is the membrane thickness [24]. $\eta_{i}$ is fixed at the physiological value of $10 \mathrm{mPa} \cdot \mathrm{s}$ [7] and $\eta_{m}$ is adjusted in the range $0.7-2 \mathrm{~Pa} \cdot \mathrm{s}$ [7]. We obtain $\theta(t), \dot{\theta}(t), \omega(t)$, and $\dot{\omega}(t)$. Time oscillations are indeed observed numerically (see [11]), whose features obtained with suitable couples of $\mu_{m}$ and $\eta_{m}$ reproduce experimental oscillations measured for both RBCs and one capsule extracted from [18], as shown in Fig. 2(b). Moreover, the model captures the trends of the $\dot{\gamma}$ variations of $\Delta \theta, T_{\mathrm{osc}}$, and the mean oscillation angle $\bar{\theta}$ as seen in the inset of Fig. 3(a). The decrease of both $\Delta \theta, T_{\text {osc }}$ reported in Fig. 3 can be directly deduced by treating the elastic contribution as a small perturbation in the second equation of (2), valid in the linear part, since one recovers the steady KS solution at the order 0 of the parameter in front of $\sin (2 \omega)$, while at the first order one finds that $\Delta \theta$ scales as $\left(\mu_{m} / \dot{\gamma}\right)$ and $\theta$ oscillates at half the TT period and is linear in $\dot{\gamma}^{-1}$. Finally, the model describes the S-T 


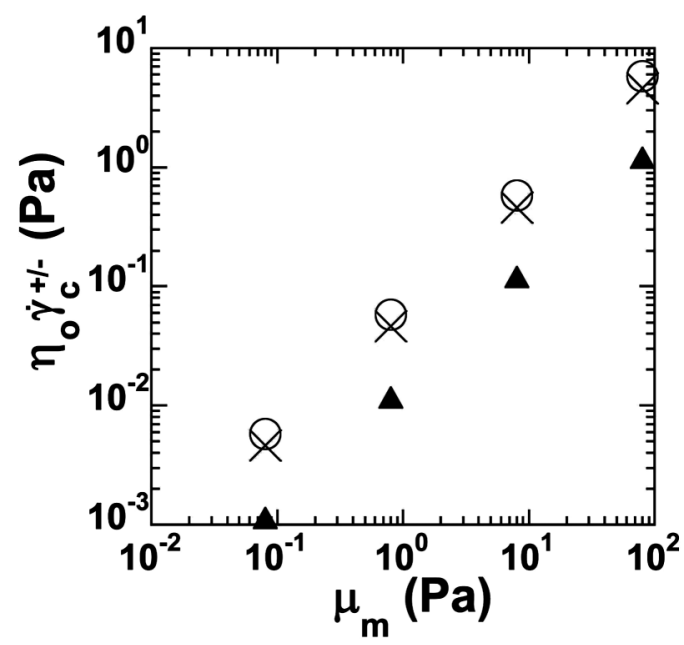

FIG. 5. Theoretical shear stresses of transition versus $\mu_{m}$ with

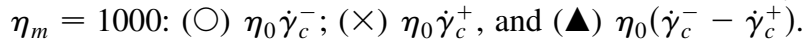

transition for decreasing values of $\dot{\gamma}$ (see [11]) and the existence of an intermittent regime as shown in Fig. 4. The theoretical $\dot{\gamma}$ domain of intermittency $\left(\left[\dot{\gamma}_{c}^{+}, \dot{\gamma}_{c}^{-}\right]\right)$has the same order of magnitude as experimental observations of $\dot{\gamma}_{c}^{>}$and $\dot{\gamma}_{c}^{<}$. As it is seen by requiring the second term in the second equation of (2) to be of the same order of magnitude as the first part, the critical shear rate should scale as $\mu_{m} / \eta_{0}$. It is indeed numerically observed (Fig. 5). Both critical values of $\eta_{0} \dot{\gamma}_{c}^{+}$and $\eta_{0} \dot{\gamma}_{c}^{-}$are mainly governed by the RBC elastic contribution for given cell geometry and may provide an average determination of $\mu_{m}$ from the observations of the transition reported in Fig. 4(b). Indeed, from Figs. 5 and 4(b), we find that $\mu_{m}$ ranges in the interval 0.14-2 $\mathrm{Pa}$. By setting the 2D shear modulus $\mu_{m, S}=\mu_{m} e$ we obtain values ranging from 0.07 to $1 \times$ $10^{-7} \mathrm{~N} / \mathrm{m}(e=50 \mathrm{~nm})$ below that usually reported [1]. We also find a comparable difference on $\mu_{m, S}$ with that reported for the elastic capsule of Fig. 2(b) from [18]. This underestimation likely originates from the major simplifications we made in order to obtain simple analytical equations allowing the full understanding of the physics of the problem: (i) simplistic constitutive equations, (ii) KS velocity field, which may overestimate membrane deformations. In particular, Tran-Son-Tay et al. [7] suggested that the Secomb-Skalak area conserving velocity field [21] would lead to a $70 \%$ increase of the membrane viscosity compared to that derived from the KS model. (iii) Treatment of strains from a 3D description of the membrane of RBCs and capsules although these systems form 2D shells [20]. However, the main interest of this tractable model is to understand the role of the various physical parameters on the motion. For example, for given external viscosity and shear rate, $\Delta \theta$ is not much sensitive to values of $\eta_{i}$ and $\eta_{m}$ taken in the physiological range. $\Delta \theta$ is essentially fixed by $\mu_{m}$, and its measurement may provide a complementary method to determine $\mu_{m}$ on individual flowing RBCs.
In conclusion, the swing and the shear-stress triggered transition of motion of RBCs show the existence of their shape memory and are a signature of their membrane shear elasticity. Despite its simplicity, our model provides a good description of the observed behavior and we believe that a more refined model should allow a sensitive determination of individual RBC mechanical properties.

We thank Dr. J. Skotheim for discussions, B. Carpentier for experimental help, Professor H. A. Stone for discussions, and Professor H. Rehage for providing us data on capsules.

*Electronic address: abkarian@lcvn.univ-montp2.fr

$\dagger$ Electronic address: viallat@marseille.inserm.fr

[1] N. Mohandas and E. Evans, Annu. Rev. Biophys. Biomol. Struct. 23, 787 (1994).

[2] S. Chien, Ann. Rev. Phys. 49, 177 (1987).

[3] T. M. Fischer, Biophys. J. 86, 3304 (2004).

[4] H. Goldsmith and J. Marlow, Proc. R. Soc. B 182, 351 (1972).

[5] T. M. Fischer and H. Schmid-Schönbein, Blood Cells 3, 351 (1977).

[6] T. Fischer, M. Stöhr-Liesen, and H. Schmid-Schönbein, Science 202, 894 (1978).

[7] R. Tran-Son-Tay, S. Sutera, and P. Rao, Biophys. J. 46, 65 (1984).

[8] S. Keller and R. Skalak, J. Fluid Mech. 120, 27 (1982).

[9] S. Sutera, P. Pierre, and G. Zahalak, Biorheology 26, 177 (1989).

[10] M. Abkarian and A. Viallat, Biophys. J. 89, 1055 (2005).

[11] See EPAPS Document No. E-PRLTAO-98-004719 for online movies and animations. For more information on EPAPS, see http://www.aip.org/pubservs/epaps.html.

[12] V. Kantsler and V. Steinberg, Phys. Rev. Lett. 96, 036001 (2006).

[13] M. Kraus, W. Wintz, U. Seifert, and R. Lipowsky, Phys. Rev. Lett. 77, 3685 (1996).

[14] H. Noguchi and G. Gompper, Phys. Rev. Lett. 93, 258102 (2004).

[15] C. Misbah, Phys. Rev. Lett. 96, 028104 (2006).

[16] P. Erni, P. Fischer, and E. Windhab, Appl. Phys. Lett. 87, 244104 (2005).

[17] K. Chang and W. Olbricht, J. Fluid Mech. 250, 609 (1993).

[18] A. Walter, H. Rehage, and H. Leonhard, Colloids Surf. A 183, 123 (2001).

[19] S. Ramanujan and C. Pozrikidis, J. Fluid Mech. 361, 117 (1998).

[20] Because of the 3D nature of the KS velocity field, the membrane deformation is considered to be 3D while other nonalgebraically tractable approaches considered the deformation field to be only 2D [7,21].

[21] T. W. Secomb and R. Skalak, Q. J. Mech. Appl. Math. 35, 233 (1982)

[22] J. M. Skotheim and T. W. Secomb (to be published).

[23] Surface area of an oblate ellipsoid: $\Sigma=2 \pi a_{1}^{2}[1+$ $\left.\left(a_{2} / a_{1}\right)^{2} \operatorname{atanh}(\mathrm{ex}) / \mathrm{ex}\right]$ with ex $=\left(1-a_{2}^{2} / a_{1}^{2}\right)^{1 / 2}$.

[24] V. Heinrich, K. Ritchie, N. Mohandas, and E. Evans, Biophys. J. 81, 1452 (2001). 